\title{
UN CASO DE CORDOMA ÓSEO (CÁNCER) EN INDIVIDUO CHANCAY DE CERRO COLORADO, SANTA MARÍA-HUAURA
}

\author{
Pieter D. van Dalen Luna ${ }^{1}$ \\ UnIVERSIDAD NACIONAL MAYOR DE SAN MARCOS \\ pvandalen2@hotmail.com \\ DayanNa L. CARBonel ARANA ${ }^{2}$ \\ UNIVERSIDAD NACIONAL MAYOR DE SAN MARCOS \\ dayannacarbonel@hotmail.com
}

\section{RESUMEN}

El presente artículo presenta el diagnóstico de un caso de cáncer óseo en un individuo humano de la cultura Chancay recuperado en el área funeraria de Cerro Colorado, extremo meridional del valle de Huaura. Cerro Colorado es un extenso complejo arqueológico conformado por varios sectores entre los que destaca: el Tambo Tawantinsuyu, el sector amurallado y la extensa área funeraria, ocupada hoy por numerosos asentamientos humanos y asociaciones de vivienda.

Palabras Claves: Arqueología, cultura Chancay, valle de Huaura, cementerio, cáncer.

\section{Abstract}

This article presents the diagnosis of a case of bone cancer in a human individual from the Chancay culture recovered in the funeral area of Cerro Colorado, southern end of the valley of Huaura. Cerro Colorado is a vast archaeological complex consisting of several sectors among which: the Tambo Tawantinsuyu, the walled sector and extensive funerary area, occupied now many human settlements and housing associations.

KeYworDs: Archeology, culture Chancay, Huaura Valley, cemetery, cancer.

1.- Arqueólogo. Docente Departamento Académico de Arqueología, Facultad de Ciencias Sociales, Universidad Nacional Mayor de San Marcos. Director del Museo de Arqueología y Antropología de San Marcos. Director del proyecto de investigación arqueológica Chancay-Huaral-Atavillos.

2.- Arqueóloga. 


\section{INTRODUCCIÓN}

Los estudios de cáncer en la arqueología andina han sido escasos debido a dos factores. El primero concierne a que el ciclo de vida del poblador andino era relativamente corto entre los 35-45 años de edad, por ello no permitiendo el desarrollo del cáncer que frecuentemente tiene un periodo de hasta 10 años de formación, siendo las edades más propensas a partir de la segunda y quinta década; y el segundo se debe a la carencia de una metodología consistente en el diagnóstico para detectar patologías de esta naturaleza (Verano \& Lombardi 1999, Altamirano \& Bueno 2013, Lastres \& Cabieses 1959, Aufderheide, et al. 1998). Por eso es necesario seguir investigando nuevas evidencias que fortalezcan la investigación del cáncer en el Perú.

En el valle de Huaura, localizado en la costa norcentral del Perú, se localizan múltiples cementerios prehispánicos en donde se han realizado estudios paleopatológicos como los realizados por Pedro Weiss (1970, 1984), Arturo Ruiz Estrada (1981), Valdivia, entre otros. En este trabajo se hará el análisis de un caso de cáncer hallado en el complejo arqueológico de Cerro Colorado en el extremo sureste del valle de Huaura.

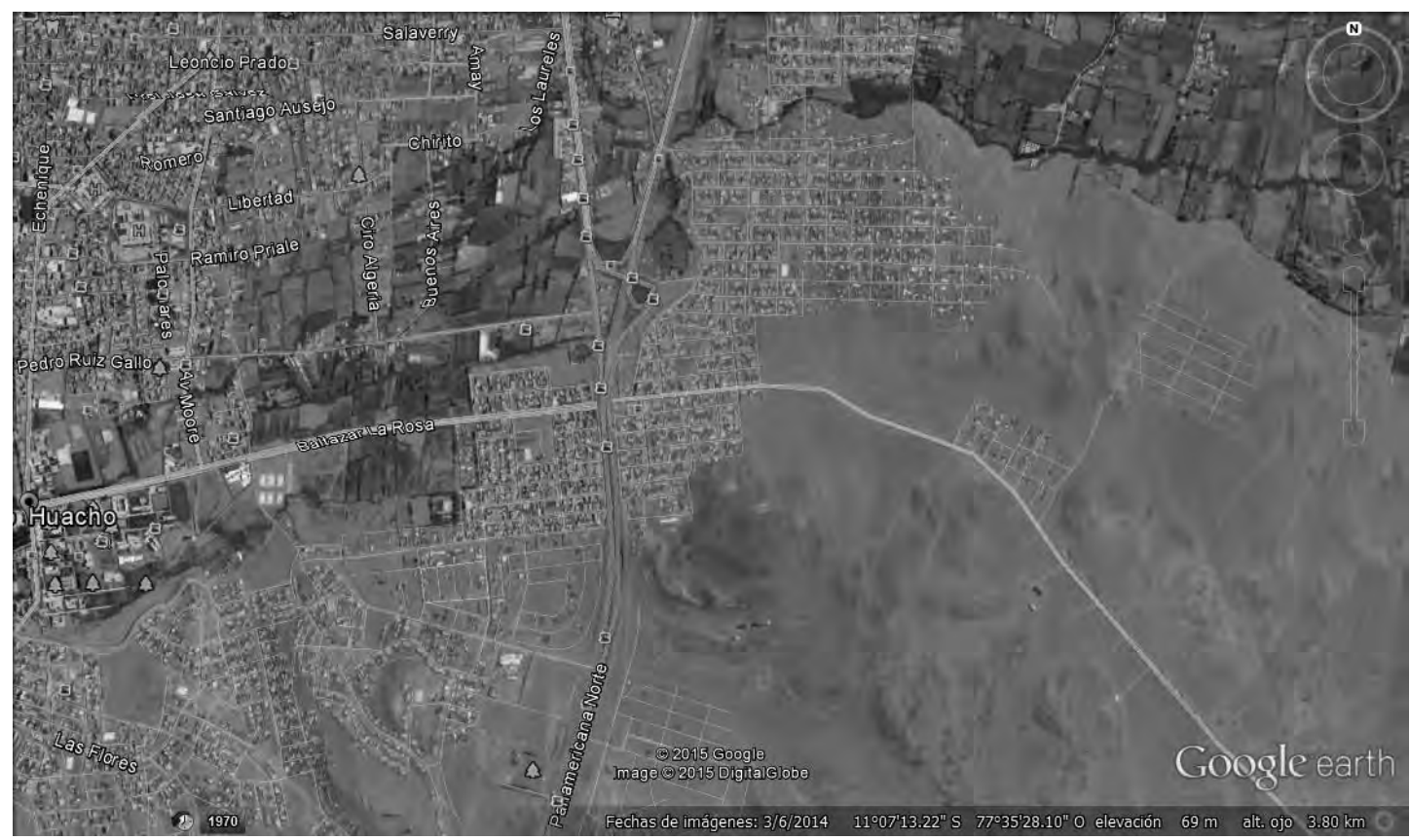

Figura 1: Vista de parte del área funeraria de Cerro Colorado con las A.V. Los Pinos, Distrito de Santa María Huaura (Google Earth), con el lugar de hallazgo del contexto funerario.

El complejo arqueológico de Cerro Colorado se encuentra ubicado en el distrito de Santa María, en la provincia de Huaura, departamento de Lima. En esta localidad se evidencia restos de ocupaciones de culturas prehispánicas como Huaura, Chancay siendo esta ultima la más influyente en toda la región inmediatamente antes del Tawantinsuyu.

\section{LOS ORIGENES DE LAS PATOLOGIAS HUMANAS}

Desde los orígenes de la humanidad hasta nuestros días, la muerte significó un enigma que el hombre no puede resolver, el miedo a lo desconocido significó un reto constante para conseguir respuestas a dicho misterio. Con el transcurso del tiempo la resignación a la muerte ya no era una 
condición que el hombre concibiese. El avance en la tecnología médica en las últimas décadas hecho que las enfermedades fueran reconocidas con nombres y tratamientos que prolongarán la vida de la población.

En tiempos de la invasión española, los cronistas de los siglos XVI y XVII (como el caso de Bernabé Cobo), describieron la forma de vida del poblador andino plasmándolo en textos y dibujos, así como las anomalías y enfermedades que sufría por males congénitos y degenerativos como producto de los cambios en la sociedad, medio ambiente, alimentación, etc. Aún se desconoce con certeza sus orígenes, sin embargo diversos autores asumen la carga genética como principal motivo de la enfermedad mientras que otros lo atribuyen a factores medioambientales o también llamados fenotípicos (Moodie 1923, Tello \& Williams 1930, Lastres \& Cabieses 1959, y Weiss 1984).

Las evidencias arqueológicas sugieren que el cáncer fue un mal que aquejó a la humanidad desde tiempos antiguos, como lo atestiguan algunas momias de Egipto, donde se han hallado evidencias de tumores óseos. En otras sociedades antiguas como la griega, también se ha reportado restos humanos con evidencias de cáncer, incluso fue el médico griego Hipócrates, quien le dio el nombre de cáncer a esta enfermedad. En Roma, el médico Celsus reportó la afectación y tratamiento de este mal. En la Edad Moderna, numerosos investigadores y médicos han reportado las características y evolución de este mal en seres humanos, mientras que en la actualidad existen grandes institutos dedicados al estudio de este mal, que es la principal causa de muerte en los seres humanos.

\section{El CONOCIMIENTO DEL CÁNCER EN LA COSTA NORCENTRAL PREHISPÁNICA}

En el presente punto se discutirá las dos hipótesis que vienen teniendo más fuerza sobre el origen del cáncer en los últimos tiempos para la ciencia médica y cuáles fueron los agentes que pudieron contribuir u ocasionar el desarrollo del cáncer en estas poblaciones costeras en periodos tempranos. Sabemos que sobre su etiología existe una amplia gama de factores, tales como los genéticos, nutricionales, ecológicos y culturales. Esto escapa a nuestra investigación debido a la carencia de frecuencia de casos antiguos y su comparación con poblaciones actuales.

1.- Hipótesis de origen genética o Genotipica: En esta línea de investigación tenemos a Knudson $(1971)^{3}$, Boveri (1914) quienes proponen que las células se transforman en células cancerosas debido a una alteración en el ADN. Las personas pueden heredar un ADN alterado, pero también las alteraciones del ADN son causadas por errores que ocurren durante la reproducción de una célula normal o por algún factor en el ambiente.

2.- Hipótesis de origen nutricional o Fenotípica: Esta hipótesis nos lleva a reflexionar sobre los hábitos alimenticios de estos pobladores y su interacción con el medio ambiente que los rodeaba, del mismo modo, como producto de un complejo número de vínculos. El fenotipo puede señalar que una persona tendrá un determinado color de piel, pero esta podrá variar de manera específica si durante la vida de la persona se ve expuesta en cantidad al sol, mientras que la piel de otra persona puede no reaccionar de la misma manera.

Las nitrosaminas han causado la atención debido a su actividad como potentes carcinógenos. Éstas se producen como consecuencia de la reacción de los nitritos con unos compuestos que forman parte de la dieta habitual: las aminas. Los nitritos, por su parte, proceden de la oxidación de los nitratos, que también se encuentran habitualmente en la dieta, (alimentos salados, carnes, pescados, etc.). En el caso en análisis, es posible que la mayor frecuencia de alimentos salados y ahumados generaron 
una faltante en el equilibrio alimenticio. Según la OMS el consumo de alimentos ahumados también elevaría la propensión a agentes cancerígenos. Su efecto tóxico proviene de los hidrocarburos policíclicos que se producen en el proceso del ahumado, impregnando elementos tóxicos en el alimento (Ramírez 2009). Datos epidemiológicos han revelado que el riesgo de desarrollar un tumor maligno presenta una gran variación entre poblaciones influenciadas en gran medida por factores ambientales y sería independiente de los factores genéticos poblacionales. Diversos estudios han demostrado la importancia de la nutrición en el desarrollo de cáncer, estimándose que hasta un 40\% de los tumores podría ser prevenido a través de la mantención de una dieta adecuada y equilibrada (Revista American Cancer Society; 2012).

La neoplasia es una alteración de la proliferación y diferenciación celular, la cual se manifiesta por la formación de una masa celular conocido como "tumor"4. El grado de crecimiento autónomo empleado por algún neoplasma puede variar ampliamente. En algunos casos, tales como el común botón óseo (osteoma), los restos de neoplasma subyacen en la mayoría por el crecimiento, regulando conjuntos en el cuerpo, $y$ formas solamente de una pequeña masa de tejido que es incapaz de destruir otras células próximas o migrando a otras partes del cuerpo. Tal comportamiento es considerado de carácter benigno. En suma, estos datos refieren a la escasez de casos de cáncer en la costa central durante los períodos tardíos y esto se debía a la carencia de investigaciones en este tema. El caso de Zapallal es el inicio de la paleopatología del cáncer en los valles del Chillón y Rímac, por lo que planteamos la hipótesis de que este mal era endémico en el pasado y confirmaría que el ciclo de vida del yunga era corto. Por eso debemos seguir hurgando en los diversos contextos arqueológicos con nuevos casos, lo que permitirá comprobar o refutar la presente hipótesis (Altamirano y Bueno; 2013).

\section{El COMPLEJO ARQUEOLÓGICO DE CERRO COLORADO}

El complejo arqueológico de Cerro Colorado se encuentra ubicado en el extremo sur del valle de Huaura, justo en el límite entre el valle mismo y las estribaciones andinas conocidas como Cerro Colorado por su tonalidad rojiza, producto de la gran cantidad de componentes férricos en proceso de oxidación que entran en contacto con la garúa estacional y los vientos. Estos cerros son pequeñas elevaciones que llegan a alcanzar los 200 metros sobre el nivel del mar. Cerro colorado es un extenso complejo arqueológico con extensas áreas funerarias, de almacenamiento y doméstico.

Por sus dimensiones y características podemos afirmar de Cerro Colorado que se constituye en el área funeraria más extensa de toda la costa central peruana, pues se extiende desde la quebrada de Pampa de Animas en el lado oriental hasta la misma línea de playa, abarcando todas las estribaciones conocidas como Cerro Colorado Grande, Cerro Colorado Chico, las Antenas, San José de Manzanares y el área de la ciudad universitaria de la Universidad Nacional José Faustino Sánchez Carrión. Así pues, esta era el área de enterramiento de los antiguos pobladores del valle de Huaura, pues mientras los grandes centros políticos administrativos y residenciales se localizaban en medio del valle, como el complejo arqueológico Walmay, Amay y La Centinela, su población era enterrada en esta gran área funeraria en medio de grandes rituales.

3.- Knudson (1971) plantea que el cáncer es el resultado de mutaciones acumuladas del ADN de las células. Esto condujo indirectamente a la identificación de los genes supresores de tumores relacionados con la progresión tumoral. Knudson realizó un análisis estadístico de los casos de retinoblastoma, para explicar precisamente el mecanismo hereditario de este tumor, como una enfermedad autosómica dominante. El retinoblastoma es un tumor de la retina que afecta por igual a niños y adultos, en niños como enfermedad genética y en adultos de forma esporádica. Los niños frecuentemente desarrollan el tumor en ambos ojos, lo que sugiere una base hereditaria predisponente. 
A pesar que casi no hay arqueólogo que no haya escuchado hablar o haya visitado este complejo de Cerro Colorado, nadie ha realizado investigación arqueológica alguna, pues solo se conocen sus materiales a partir de proyectos de evaluación y rescates arqueológicos (Tosso 1997, Rodríguez 2007, Morales 2009, van Dalen, et al 2014) o por la acción del huaqueo. Las únicas investigaciones desarrolladas hasta la fecha en este importante sitio arqueológico son los realizados por Arturo Ruiz Estrada (1981, 1991, 1998) y van Dalen $(2004,2008)$.

El área más importante se encuentra ubicado hacia el lado este de la carretera Panamericana, inmediatamente al ingresar a la ciudad de Huacho, terreno que hoy se encuentra ocupado por numerosos asentamientos humanos como es el caso de: Alberto Fujimori Fujimori, Los Pinos, San Antonio, Las Palmeras Unidas y Cerro Colorado, los cuales cada año van avanzando y ocupando parte del cementerio huaqueado.

Esta área funeraria está conformada por numerosos contextos funerarios disturbados característicos de la cultura Chancay, entidad sociopolítica que se desarrolló en el Intermedio Tardío (1000 - 1470 d.c.) entre los valles de Chancay y Huaura (van Dalen 2008, 2012), observándose en superficie abundante material cerámico del estilo Chancay en sus tipos Negro sobre Blanco, Tricolor y Pasta roja; así como estilos del Horizonte Medio (Teatino, Huaura, Supe, Pativilca, etc.). Se aprecian además abundantes retazos de textiles que conformaban los fardos, de naturaleza llana o listados en color marrón sobre fondo crema, muy similar a la decoración de la cerámica.

Esta área presenta también una edificación ortogonal de planta cuadrangular que corresponde a un tambo del periodo Tawantinsuyu. Cabe señalar que en la parte superior hay un cerro con tres sistemas de murallas concéntricas.

Durante el desarrollo del análisis forense realizado entre junio y setiembre de 2014 a 70 contextos humanos en el proyecto arqueológico Cerro Colorado, se pudo evidenciar la alta mortandad de individuos de sexo femenino en edades reproductivas (20-35 años) tiene un rango más frecuente, así como también la concurrencia de niños y bebes en etapa de lactancia. La presencia de individuos masculinos es baja.

Las patologías más frecuentes son: descalcificación, infecciones dentales, anemia criba orbitalia, espondilolisis, enfermedades degenerativas como la artrosis, malformación en las cabeza de fémures y caderas, osteomas y tumores y otras producto de las actividades físicas realizadas como hipervascularización, exostosis del conducto auditivo, etc.

\section{UN CONTEXTO FUNERARIO CON CÁNCER ÓSEO}

En esta parte del artículo vamos a presentar las características de un contexo funerario recuperado durante las excavaciones del sector funerario del complejo arqueológico de Cerro Colorado, en el área superpuesta por la Asociación de Vivienda Los Pinos IV Etapa. El contexto funerario se distingue de los demás hallados contiguamente, debido a que el individuo presenta un caso de cáncer óseo, lo cual le ocasionó la muerte.

4.- Los tumores son una masa anormal de tejido, producida por la multiplicación de algún tipo de células; esta multiplicación es descoordinada con los mecanismos que controlan la multiplicación celular en el organismo, y los supera. Además, estos tumores, una vez originados, continúan creciendo aunque dejen de actuar las causas que los provocan. La neoplasia se conoce en general con el nombre de cáncer. El origen de esta palabra se remonta a Ambroise Paré en 1517 (http://escuela.med.puc.cl/ publ/patologiageneral/Patol_090.HTML). 


\section{La unidad de excavación 18}

Entre los años 2014 y 2015 se ha venido desarrollando trabajos de excavación intensiva en amplias unidades de rescate a fin de recuperar la totalidad de contextos funerarios existentes en las calles y avenidas.

En la etapa IV de la Asociación de Vivienda Los Pinos (Ex A. H. Alberto Fujimori Fujimori), en la calle Independencia, esquina nor-oeste del parque Las Gardenias, se emplazó la unidad de excavación 18, con dimensiones: de norte a sur 10.48 metros, de este a oeste 15.88 metros, de sur a norte 11.09 metros y de oeste a este 16.66 metros. El terreno es irregular con una ligera pendiente, de este a oeste. La unidad comprende en total 6 subunidades.

La capa A es un relleno cultural de color marrón rojizo, de entre 0.17 y 0.32 metros de grosor, sin evidencias de material moderno, conformada por componentes naturales como arena, piedras pequeñas, raíces, abundante material cultural prehispánico, en gran porcentaje fragmentos de cerámica Chancay (bordes, bases, asas, fragmentos del cuerpo), material textil, botánico (maíz, algodón, lagenarias), restos de artefactos como soguillas (junco), material malacológico y restos de carbón. Presenta una textura gruesa, consistencia semicompacta debido al uso agrícola del espacio función, que se le dio con anterioridad (filtración de agua). La distribución de la capa es homogénea por toda la unidad.

La capa B por su parte, es de color beige y de un grosor de entre 1.70 y 1.50 metros, estaba conformado por arena gruesa con pequeñas y medianas piedras con formas angulosas; en algunos perfiles

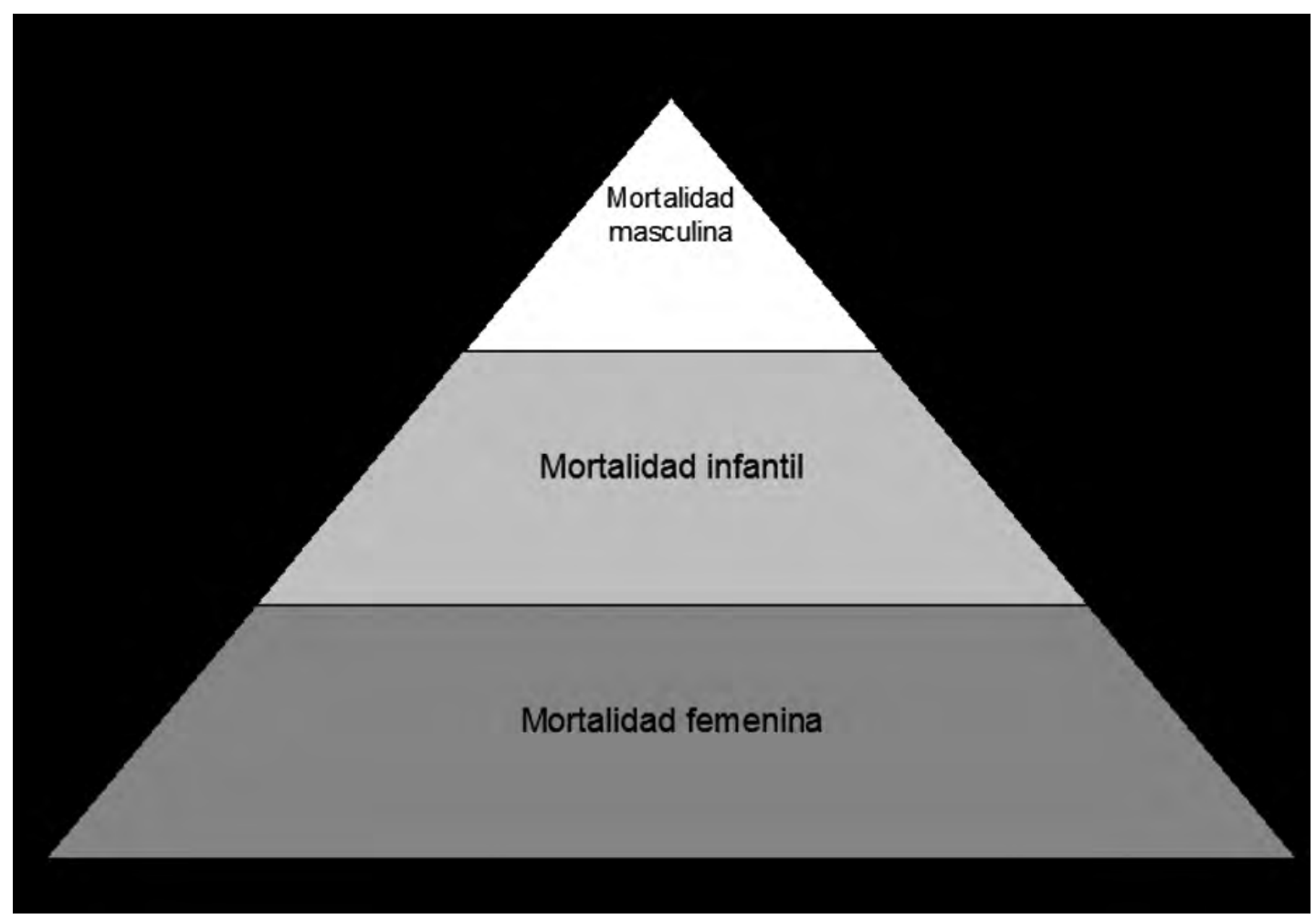

Figura 2: Pirámide estadística de mortalidad en el A.A.H.H. los Pinos - Huaura durante el Periodo Intermedio Tardío. 
de la unidad se nota mayor concentración de ripio, así como también lentes finos de arcilla natural. Este es un estrato de origen natural, en cuanto a su consistencia es suelta, sin embargo en algunas zonas se vuelve más compacta. Se hallaron en esta capa 9 contextos funerarios.

\section{Descripción del contexto funerario:}

El contexto funerario estudiado fue denominado como Contexto Funerario 2, ubicado en la unidad 18, Capa B. Se trata de un individuo adulto, de sexo masculino, la posición en la que se encontraba el cuerpo es flexionado en decúbito dorsal, extremidades superiores flexionadas, manos extendidas hacia la mandíbula inferior, extremidades inferiores flexionados hasta aproximadamente la mitad de las costillas. No tenía ningún material asociado. Los restos del individuo están incompletos, ausencia de la tibia, peroné, pie. Se observa el sacro y la última vértebra lumbar fusionadas, produciéndose un abultamiento muy notorio. No presenta material asociado ni textil que lo cubra (Figura 4).

Para la determinación del género se aplicó los métodos de reconocimiento a partir del análisis de los arcos superciliares robustos, glabela pronunciada, malares sobresalientes, apófisis mastoidea notable, arcos zigomáticos fuertes, espina nasal elevada; los cuales determinaron que el sexo del individuo era masculino (Bass 1971, 1986, Comas 1976). Así mismo en las extremidades superiores manifiesta engrosamiento y rugosidad en la región de la tuberosidad deltoidea producida por actividades físicas frecuentes. En los fémures la hipervascularización es fuerte en la parte proximal.

\section{Características del cráneo}

Entre las características encontradas hallamos evidencia de deformación craneana de tipo tabular oblicuo izquierdo y fronto-occipital; en el esplagnocráneo observamos el tabique desviado hacia la derecha y microfracturas en la región, posiblemente debido a golpes que muestran las secuelas de tiempos de conflictos. En la cavidad bucal las infecciones son severas, tanto que en algunos casos han causado la pérdida de piezas dentales a temprana edad, lo cual lo manifiesta con las obliteraciones en los molares e incisivos. La alimentación es un factor determinante que causó el desgaste dental, en este caso específico muy severo, ya que en algunos casos ha perdido el esmalte y se nota expuesta la dentina como producto del consumo de alimentos duros y semillas. El consumo de la hoja de coca también era muy frecuente.

\section{Descripción paleopatológica del abdomen.}

Del análisis resaltan múltiples patologías entre ellas las que están presentes en las lumbares (L3, L4, L5) con un cuadro de espondilolisis como producto de los constantes esfuerzos físicos cargando peso, formando pequeñas espículas de callos óseos en los bordes anteriores y posteriores de los cuerpos vertebrales. Estas formaciones son producto de las intensas actividades degenerativas de los osteocitos y osteoblastos, patología recurrente en los pescadores y agricultores.

En la columna vertebral muestra lesiones porosas y aplastamiento del cuerpo vertebral predominantemente en la zona torácica y lumbar. En las apófisis transversales presenta labiamientos en toda la columna vertebral. En la zona lumbar la presencia de espondilolisis es severa con crecimiento del borde del cuerpo vertebral (Figura 6).

Las lesiones encontradas en el sacro y en la última vértebra lumbar (L5), en su cara ventral del cuerpo vertebral, muestra un tumor óseo o cordoma, el cual es un tumor benigno con altas posibilidades de tomar una reacción agresiva, que mide 6 x $4 \mathrm{~mm}$, de forma oval y orientada hacia la derecha. La superficie del tejido óseo es ligeramente rugoso con ondulaciones alisadas. Este osteoma habría causado presiones a los órganos de los intestinos delgados e inicio de los gruesos. Es posible que el 
crecimiento del tejido óseo haya ocurrido en edad adulto-joven, de forma indolora, y posteriormente al paso de su desarrollo manifestar dolores pélvicos, presión en las piernas, calambres y dolores de los miembros inferiores.

En la cara ventral del sacro aparecen lesiones líticas ${ }^{5}$ diseminadas y próximas a esta área articular con bordes irregulares. Asimismo, presenta dos severas lesiones líticas perforantes con reacción ósea de 6 × $2 \mathrm{~mm}$ (lado derecho); otro de $65 \times 22 \mathrm{~mm}$ en la cara ventral (lado izquierdo), alcanzando hasta la cara dorsal. Las porosidades que muestra en la cara vertebral son de formas circulares, de aspecto rugoso en los bordes y espículas óseas que sobresalen. Las porosidades son constantes y conocidas como lesiones osteolíticas o líticas y van destruyendo de la cara vertebral hacia la cara dorsal. La lesión se proyecta afectando los órganos blandos contenidos en esta cavidad como por ejemplo: los intestinos gruesos, el recto, la vejiga, posiblemente la próstata y colon, entre otros (Zimmerman \& Kelley 1982, Rotshchild, et al. 1998).

\section{Diagnóstico diferencial}

El presente caso expone doble forma de afectación cancerosa: en una el cordoma de gran magnitud se ha producido en la columna vertebral, debido a que existe restos de notocorda ${ }^{6}$, que se constituye en un alto porcentaje de los tumores óseos primarios, del cual su origen es controversial; y la otra es la lesión lítica destructiva. El cáncer óseo es una neoplasia devastadora y con mayor incidencia en hombres ${ }^{7}$. Este es el proceso destructivo final de la enfermedad.

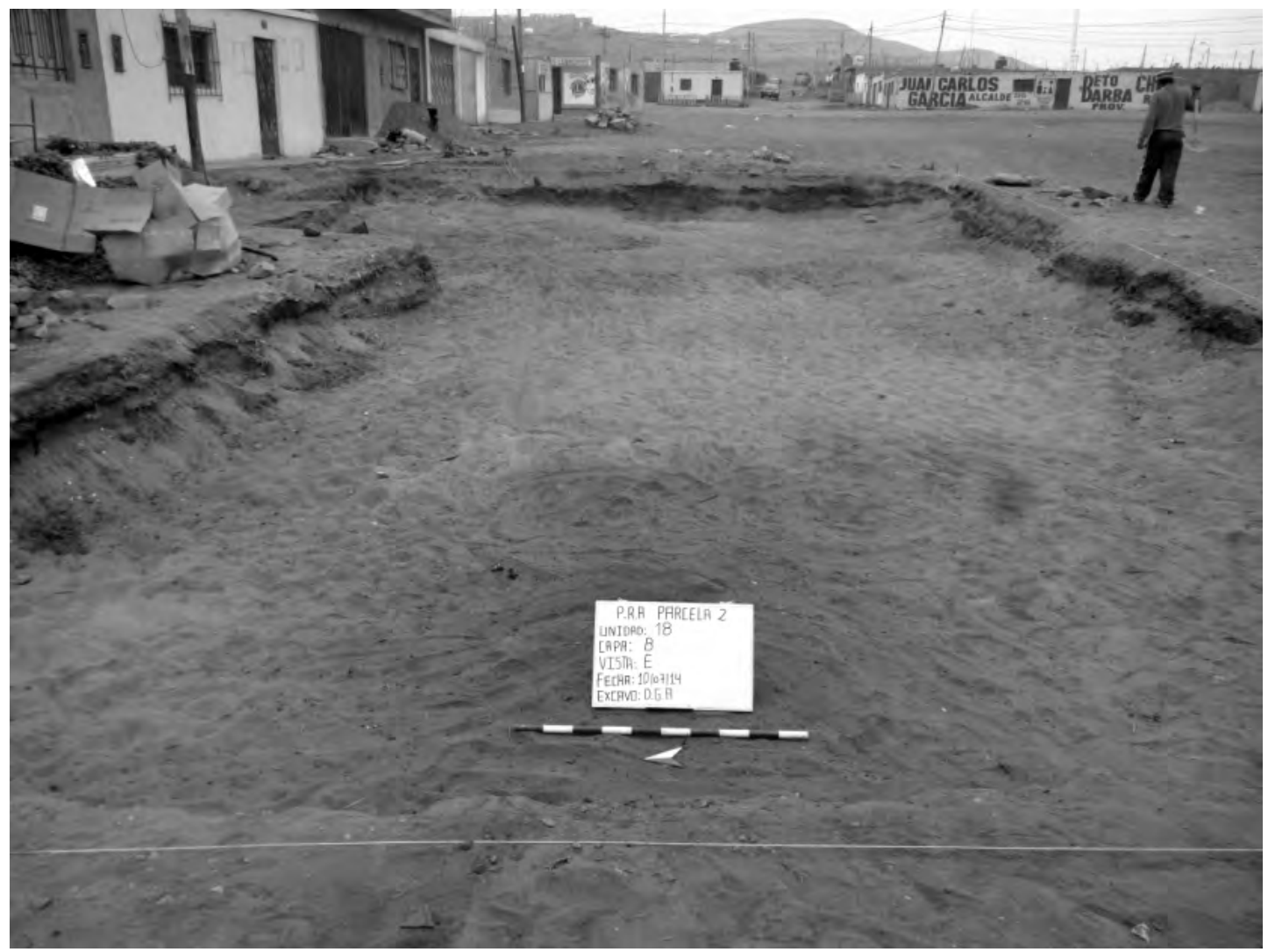

Figura 3: Vista panorámica de la unidad de excavación 18, parcela 2, Cerro Colorado. Excavación en área de la capa B, donde aparece el caso bajo estudio. 


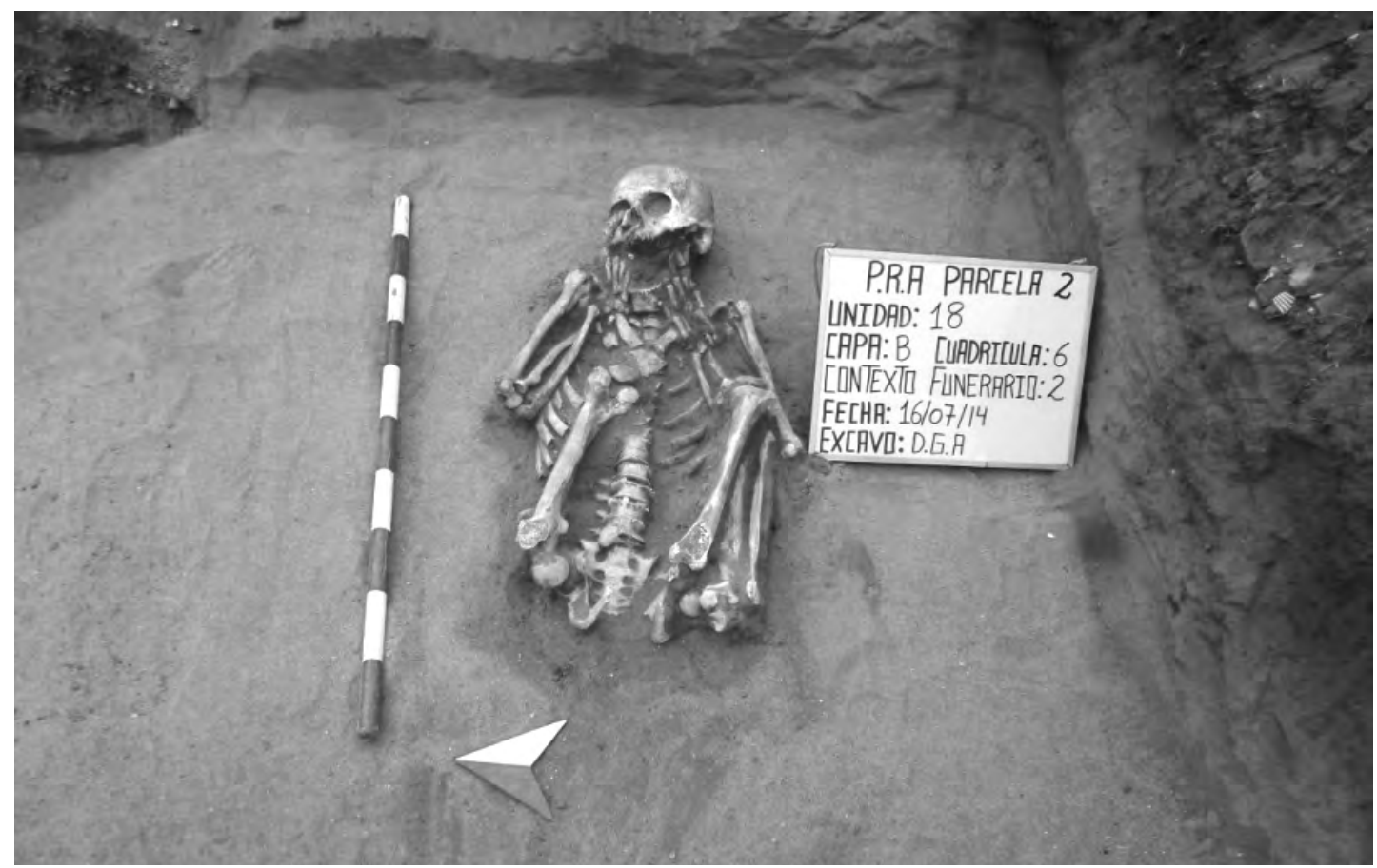

Figura 4: Entierro primario, en posición decúbito dorsal, brazos y piernas flexionadas. Vista de planta del contexto funerario № 2 .

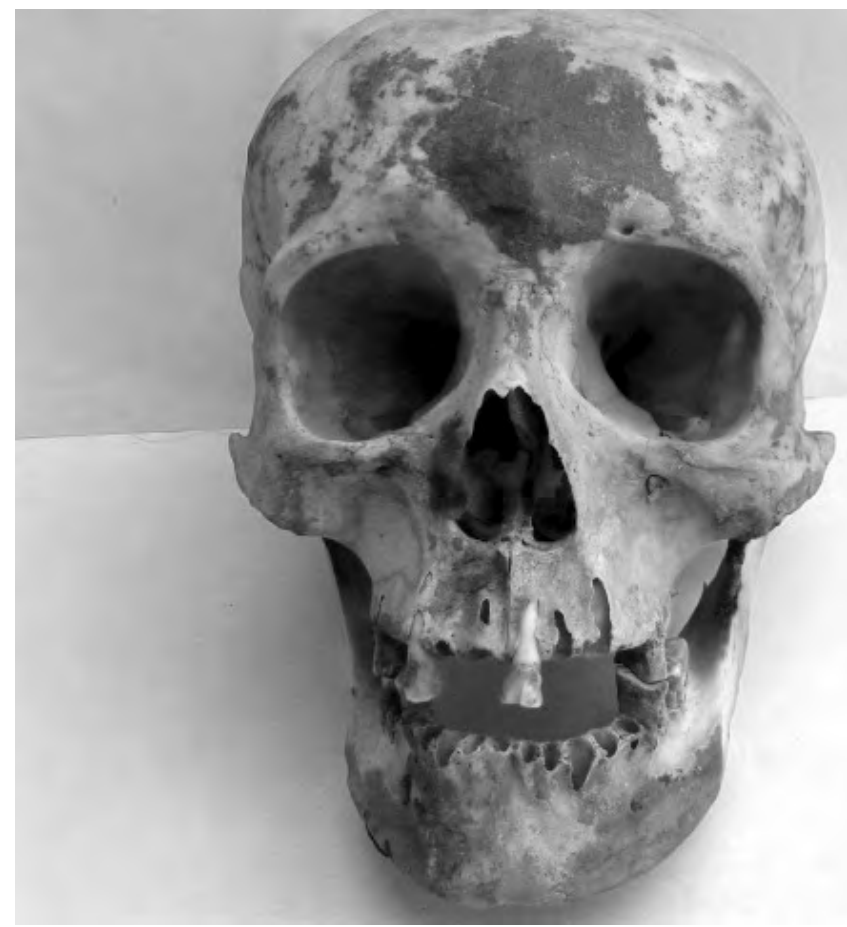


Asimismo, otros tipos de cáncer como: el mieloma múltiple, de mama, próstata y pulmón, también tienen alta posibilidad y prognosis a ser metástasis (Coleman; 1997). Sin embargo, en el caso que exponemos, no llegó a desarrollarse la metástasis, el individuo de edad adulta joven fue afectado gravemente por ambos tipos de cáncer localizados en la región sacro-lumbar y sacro-coccígea (Ortner \& Putschar; 1985, Aufderheide \& Rodríguez 1998).

Los cordomas frecuentemente se localizan en la zona sacro-coccígea, pero su origen puede comenzar en las partes blandas o generalmente muy cerca de esta región, afectando las láminas interna y externa del sacro. Luego se expande interrumpiendo e invadiendo tejidos blandos adyacentes como los intestinos, la vejiga y el recto. (Coley 1960, Aufderheide \& Rodríguez 1998)

Es posible que el individuo en análisis haya padecido de cáncer a un tejido blando (no visible en la actualidad) en la región sub-abdominal, para luego trasladarse a los huesos creando su expansión destructiva. Los cordomas durante su crecimiento son indoloros y su desarrollo varía entre 1 a 5 años (Masson; 1999), es decir tiene una evolución lenta pero progresiva. Posteriormente, los dolores de cadera se acrecientan creando molestias en la persona que la padece. En ocasiones el déficit motor progresa hasta limitar cada vez mas las actividades diarias, sumándose los problemas de incontinencia vesical y rectal, e incluso cuando el crecimiento es mayor puede crear destrucción intestinal.

El análisis paleopatológico demuestra que el individuo se encontraba en un estado de desnutrición fuerte, posiblemente producto de lo desgastante de la enfermedad. Todo ello ayudó a agilizar el

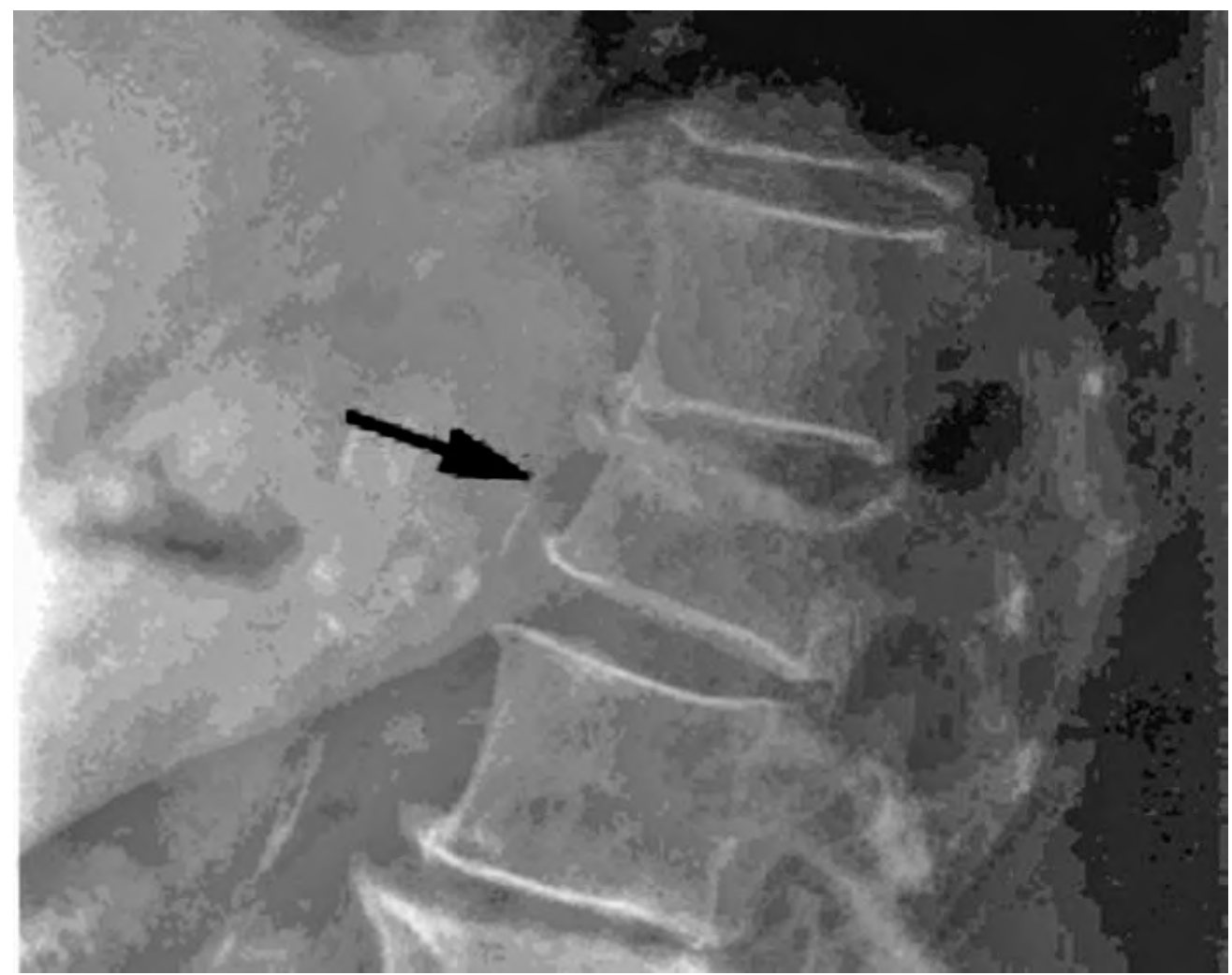

Figura 6: Radiografía en que se muestra un caso similar al de nuestro estudio, con el aplastamiento de las vértebras lumbares (L3, L4 y L5) y espondilolisis. 
deceso. Debido a que las células cancerosas activan los osteoclastos, el hueso comienza un proceso de desgaste sin regeneración de tejidos óseos nuevos (Brown, Cook, Major 2005, Lipton 2006).

Se han registrado pocos casos de cáncer arqueológico en el Perú, posiblemente debido a la escasa investigación en dicho tema en individuos del antiguo Perú y/o por mayor incidencia de tipos de cáncer que afectaba a tejidos blandos, con lo que resulta más difícil su identificación.

\section{Conclusiones}

El presente caso muestra claras evidencias de cordoma, ya que esta patología se manifiesta principalmente en la parte superior del sacro. Este caso analizado era frecuente en poblaciones agrícolas que tendrían -como hipótesis- dos causas: la primera, la utilización de metales pesados como pinturas faciales o corporales (tatuajes); y la segunda, al consumo de aguas con alta concentración de minerales pesados como el cobre arsenical. Este es el primer caso de un individuo con cordoma hallado en el antiguo Perú, correspondiente a la cultura Chancay. El complejo arqueológico de Cerro Colorado fue una extensa área funeraria de la cultura Chancay que alberga los restos de miles de individuos, cuyas causas de muerte fueron múltiples.
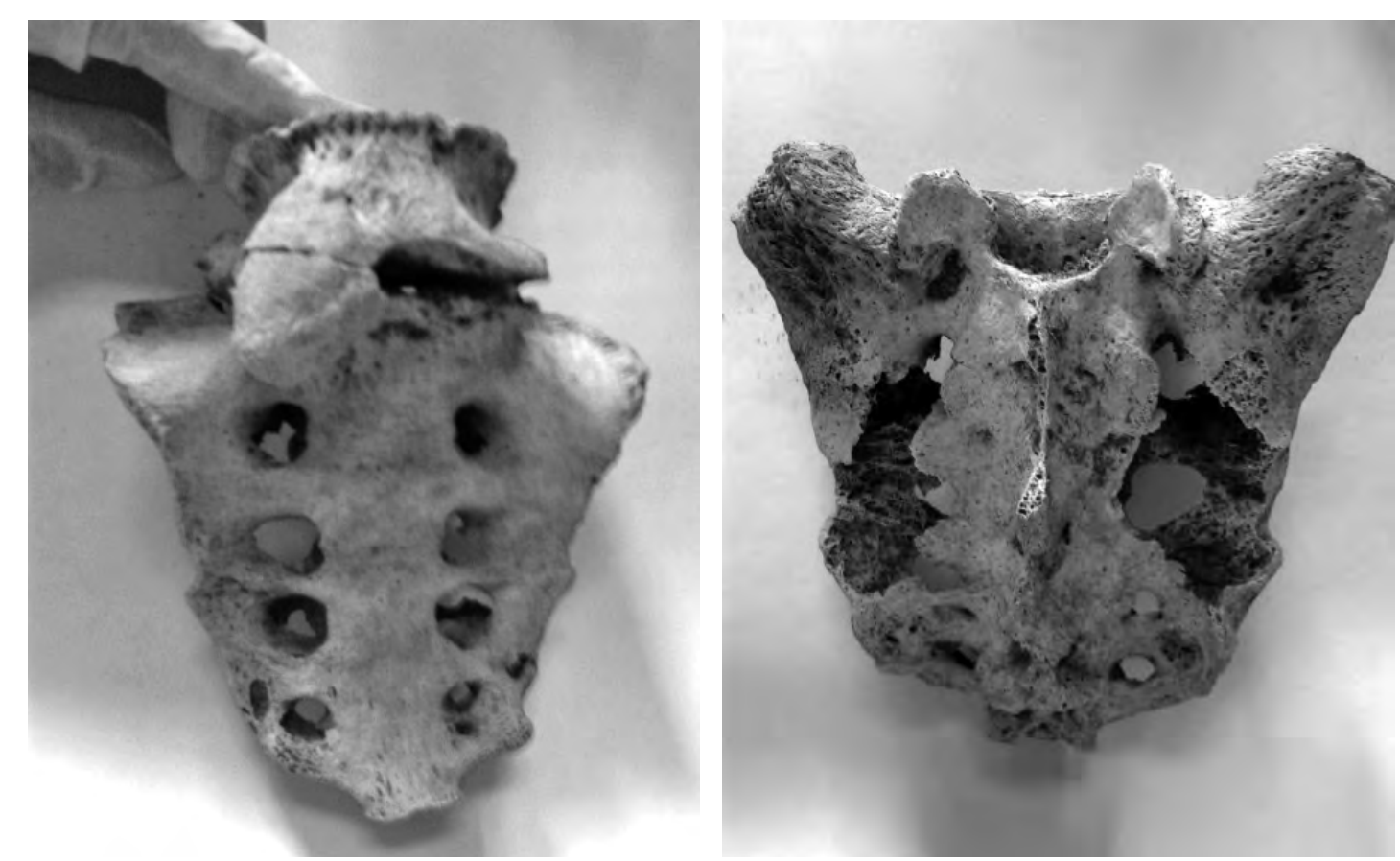

Figura 7 (izquierda): Cordoma fusionando el sacro y la última vértebra producto del crecimiento del tumor y propagándose hacia la cara ventral del sacro. Figura 8 (derecha): Cara vertebral del sacro evidencia dos lesiones líticas como producto del desarrollo del cáncer óseo.

5.- Una lesión lítica es una perforación del tejido óseo en forma circular u oval, con rebordes porosos y espículas. 6.- Conducto notocordal: es un cordón de células macizas que van a evolucionar hacia la formación de la placa notocordal. En esta estructura podemos empezar a ver la fusión de la pared ventral del ectodermo primitivo con dicho conducto. También se conoce como conducto entérico.

7.- El osteosarcoma es más frecuente en varones, quizá debido a la mayor duración en ellos de la fase de crecimiento del esqueleto comparado con la de las mujeres (Dorfman 1998, Sullca et al. 2015). 


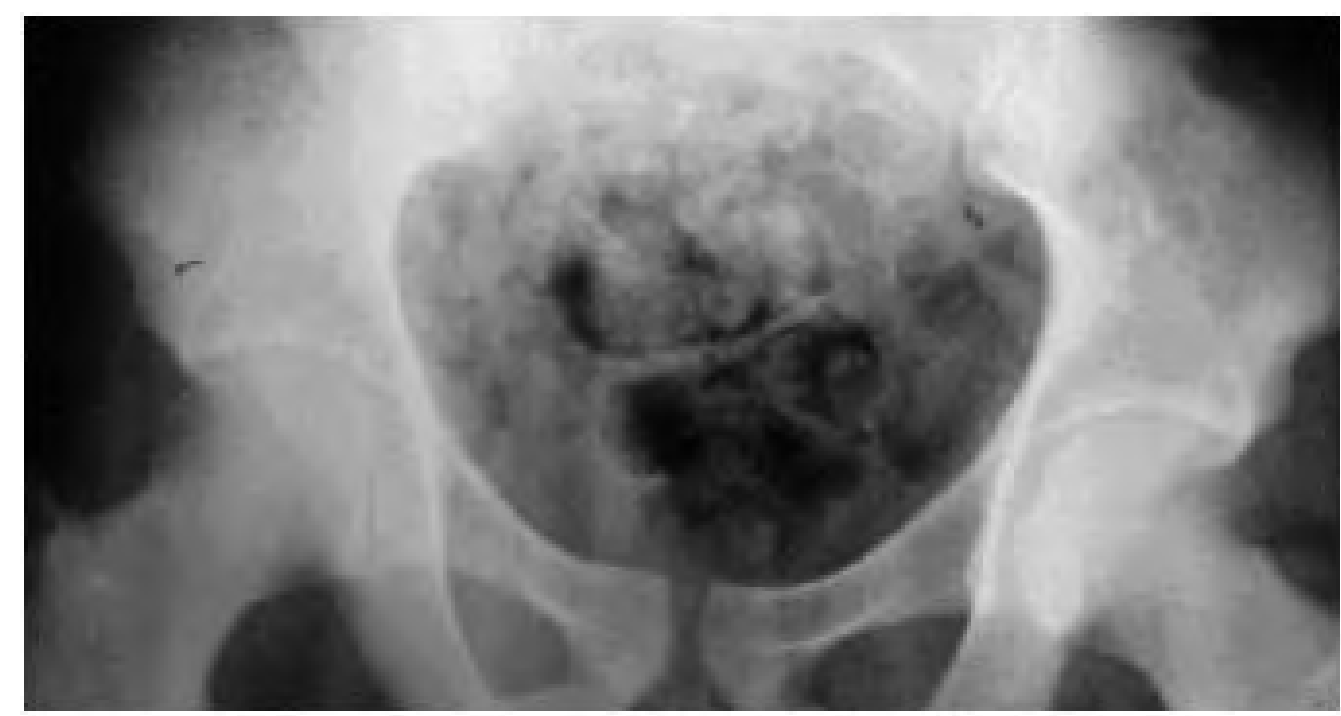

Figura 8: radiografía de un sacro con cordoma y evolución del tumor canceroso en la última vértebra lumbar, a modo de comparación.

\section{Agradecimiento}

Un especial agradecimiento al Dr. Alfredo Altamirano por las orientaciones científicas para la realización del presente artículo.

\section{BiBLIOGRAFÍA}

\section{ALTAMIRANO ENCISO, Alfredo y BUENO MENDOZA, Alberto}

2013 "Un caso de cáncer metastásico en Zapallal a fines del Horizonte Medio (1100-1200 d.C.), valle del Chillón, Perú". Investigaciones sociales. Instituto de Investigaciones Sociales, Facultad de Ciencias Sociales, Universidad Nacional Mayor de San Marcos. №30. Lima, pp. 91-104.

ANDERSON, T., WAKELY, J. \& CARTER, A.R.

1992 "Medieval example of Metastatic carcinoma: a dry bone, radiological and SEM studies". American Journal of Physical Anthropology, 89: 309-23.

\section{AUFDERHEIDE, A. \& C. RODRIGUEZ-MARTIN}

1998 Human Paleopathology. The Cambridge Encyclopedia, Cambridge University Press, UK.

\section{BASS, W.M.}

1971 Human Osteology: A Laboratory and Field Manual of the Human Skeleton. Special Publications, Missouri Archaeology Society, Special Publication 2, Columbia, Missouri.

BOVERI, Theodor

1914 Concerning the origin of malignant tumours. Cold Spring Harbor Laboratory Press.

BUIKSTRA, J.E. \& UBELAKER, D.

1994 Standards for data collection from human skeletal remains. Arkansas: Arkansas Archaeological Survey Press. 
BROWN, J.E., COOK, R.J., MAJOR, P.,

2008 Bone turnover markers as predictors of skeletal complications in prostate cancer, lung cancer, and other solid tumors. J Natl Cancer Inst. 49:565-570

COLEMAN, Re.

1997 “Skeletal complications of malignancy”. En: Cancer. № 80. Lima, pp. 1588- 1594.

COMAS, Juan

1976 Manual de Antropología Física. Universidad Autónoma de México, México. 710 pgs.

DORFMAN H.D., CZERNIAK B.

1998 “Osteosarcoma”. En: Bone Tumors. St. Louis, pp. 128-252.

DOSNE PASQUALINI, Christiane

2012 EDITORIAL MEDICINA (Buenos Aires) 72: 530-532 “Oncología experimental Volviendo a Boveri y Virchow" № 72 - № 6,

GARCÍA BARRENO, Pedro

2008 "El legado de Hipócrates" los grandes temas de la medicina. Madrid- España

KNUDZON, Alfred.

1971 "Mutation and cancer: statistical study of retinoblastoma". En: Proc Natl Academy Science U S A. № 68 (4). Washington, pp. 820-823.

LASTRES, Juan B. \& CABIESES, Fernando

1959 “La Trepanación del cráneo en el Antiguo Perú”. En: Anales de la Facultad de Medicina, № 42 (3). Universidad Nacional Mayor de San Marcos. Lima, pp. 258-320.

MOODIE, Roy

1923 Paleopathology. An introduction to the study of ancient evidences of Disease. Urbana, IL: University of Illinois Press.

MORALES CAUTI, Héctor

2009 Informe final del Proyecto de rescate Arqueológico Complementario en la Parcela 3, sitio arqueológico de Cerro Colorado, Áreas Ocupadas II, III, IV y V, Asociaciones de Vivienda Palmeras Unidas y Cerro Colorado, distrito de Santa María, Provincia de Huaura. Informe final presentado al INC.

ORTNER, Don J. \& AUFDERHEIDE, A.C.

1991 Paleopathology: Current Syntesis and Future Options. Washington, D.C.: Smitshsonian Institution, (Orgs.).

ORTNER, Don J. \& PUTSCHAR, W.G.J.

1985 Identification of Pathological Condition in Human Skeleton Remains. Washington D.C.: Smithsonian Institution Press.

RAMÍREZ OVIEDO, Pablo

2009 “Influencia de los Nitritos y los Nitratos de la dieta de Cáncer Gástrico". En: Revista Medica de Costa Rica y Centroamérica, N LXVI (589). San José, pp. 275-277 


\section{RODRIGUEZ GUILLÉN, Luis}

2007 Informe final del Proyecto de Rescate Arqueológico en la Parcela 3 del sitio Cerro Colorado - Huacho. Informe final presentado al Instituto Nacional de Cultura. 124 pp. más planos.

RUIZ ESTRADA, Arturo.

1981 "Reconocimientos Arqueológicos en Cerro Colorado (Huacho)". CICITEH Boletín del Centro de Investigación de Ciencia y Tecnología No. 01. Universidad Nacional José Faustino Sánchez Carrión. Huacho, 14 pp.

1991 "El entierro de un músico Prehispánico en Huacho, valle de Huaura" En: Estudios sobre la cultura Chancay, Perú. Krakow., Polonia, Karkow, pp. 133 - 153

1998 “Sobre el hallazgo de momias tatuadas en Huacho". En: Revista INSULA, № 4, Febrero. Huacho, pp. 6-7.

SULLCA, Arelí; TAIRA, Johnny y ALTAMIRANO, Alfredo

2015 “El tejedor: un caso de cancer metastásico en l Huaca Potosí, valle bajo del Rímac". En Prensa en el presente volumen.

TELLO, Julio C. \& WILLIAMS, Herberth

1930 "An ancient syphilis skull from Paracas in Peru". En: Annals of medical history. New series. Vol. 2, № 5. New York, pp. 515-529.

TOSSO MORALES, Walter

2000 Informe final del Proyecto de Estudio de Evaluación Arqueológica en la modalidad de evaluación, Prospección y Reconocimiento sistemático con excavaciones en el Asentamiento Humano Alberto Fujimori entregado al INC. Informe final presentado al INC.

VAN DALEN LUNA, Pieter

2004 "Los valles de Huaura y Chancay dentro del imperio del Tahuantinsuyo" En: Boletín del Patronato de defensa del patrimonio cultural del valle de Huaura y Ambar. № 16, año III, Junio. Huacho, pp. $3-8$.

2008 Los ecosistemas arqueológicos en la cuenca del río Chancay - Huaral. Su importancia para el desarrollo de las formaciones sociales prehispánicas. Ed. Gutemberg. Lima, 185 pp.

2011 “El Tawantinsuyu en la costa norcentral peruana: valles de Chancay y Huaura” Investigaciones Sociales, № 27. Instituto de Investigaciones Histórico Sociales. Facultad de Ciencias Sociales. Universidad Nacional Mayor de San Marcos. Lima, pp. 77-104.

2012 “Arqueología tardía del valle Chancay-Huaral: Identificando la nación Chancay" Investigaciones sociales. $\mathrm{N}^{\circ}$ 28. Instituto de Investigaciones Sociales, Facultad de Ciencias Sociales. Universidad Nacional Mayor de San Marcos. Lima, pp. 271-284.

VAN DALEN LUNA, Pieter; GRADOS RODRIGUEZ, Hans; TELLO CUADROS, Roberto; FLORES LIVIA, Wendy; VIVANCO RAMOS, Iván; MARCELO GONZÁLES, Yerovi

2014 "Resultados del proyecto de rescate arqueológico en el área de la parcela 4 y 5 del sitio arqueológico Cerro Colorado, Santa María, provincia de Huaura" Kullpi. Investigaciones culturales en la provincia de Huaral y el Norte Chico. Año 7, No 7, Lima, pp. 189-216. 
VERANO, John y LOMBARDI, Guido

1999 “Paleopatología en Sudamérica andina" En: Bulletin del'Institut français d'études andines, № 28 (1). Lima, pp. 91-121.

WEISS, Pedro

1970 “Introducción a la Paleopatología Americana". En: Texto de Patología, La Prensa Médica Mexicana, Editorial Fournier, México D.F.

1984 “Paleopatología Americana”. En: Boletín de Lima. № 33. Lima, pp.17-52.

Zimmerman \& Kelley; 1982,

\section{REFERENCIAS:}

2014 American Cancer Society

http://www.cancer.org/acs/groups/cid/documents/webcontent/002284-pdf.pdf

La etimología del cáncer y su curioso curso histórico "Revista Peruana de Medicina Experimental y Salud Publica” versión impresa ISSN 1726-463. Rev. perú. med. exp. salud publica vol.30 no.1 Lima ene. 2013

Guías de la Sociedad Americana contra El Cáncer sobre nutrición y actividad física para la prevención del cáncer Last Medical Review: 1/23/2012 Last Revised: 1/23/2012 2012 American Cancer Society 
